\title{
ACCOUNTS
}

of chemical research

\section{Binuclear Metallohydrolases: Complex Mechanistic Strategies for a Simple Chemical Reaction}

\author{
GERHARD SCHENK, ${ }^{*,+}, \neq$ NATAŠA MITIĆ, \\ LAWRENCE R. GAHAN, ${ }^{\dagger}$ DAVID L. OLLIS, ${ }^{\S}$ ROSS P. MCGEARY, ${ }^{\dagger}$ \\ AND LUKE W. GUDDAT ${ }^{\dagger}$ \\ ${ }^{\dagger}$ School of Chemistry and Molecular Biosciences, The University of Queensland, \\ Brisbane, QLD 4072, Australia, ${ }^{\ddagger}$ Department of Chemistry, National University \\ of Ireland -Maynooth, Maynooth, County Kildare, Ireland, and \\ ${ }^{\S}$ Research School of Chemistry, Australian National University, Canberra, \\ ACT 0200, Australia
}

RECEIVED ON MARCH 6, 2012

\section{CONSPECTUS}

B inuclear metallohydrolases are a large family of enzymes that require two closely spaced transition metal ions to carry out a plethora of hydrolytic reactions. Representatives include purple acid phosphatases (PAPs), enzymes that play a role in bone metabolism and are the only member of this family with a heterovalent binuclear center in the active form $\left(\mathrm{Fe}^{3+}-\mathrm{M}^{2+}, \mathrm{M}=\mathrm{Fe}, \mathrm{Zn}, \mathrm{Mn}\right)$. Other members of this family are urease, which contains a di- $\mathrm{Ni}^{2+}$ center and catalyzes the breakdown of urea, arginase, which contains a di- $\mathrm{Mn}^{2+}$ center and catalyzes the final step in the urea cycle, and the metallo- $\boldsymbol{\beta}$-lactamases, which contain a di- $\mathrm{Zn}^{2+}$ center and are virulence factors contributing to the spread of antibiotic-resistant pathogens.

Binuclear metallohydrolases catalyze numerous vital reactions and are potential targets of drugs against a wide variety of human disorders

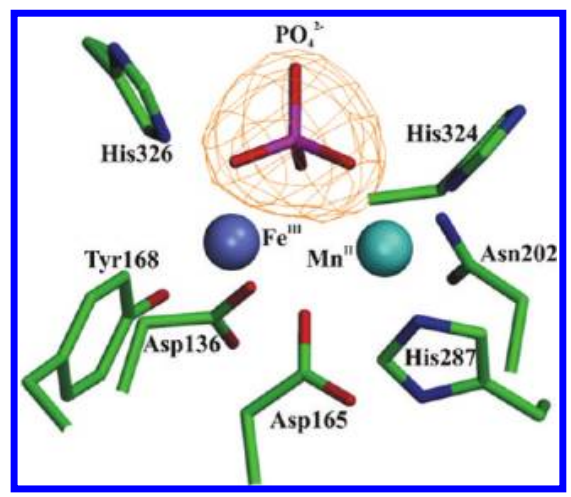
including osteoporosis, various cancers, antibiotic resistance, and erectile dysfunctions. These enzymes also tend to catalyze more than one reaction. An example is an organophosphate (OP)-degrading enzyme from Enterobacter aerogenes (GpdQ). Although GpdQ is part of a pathway that is used by bacteria to degrade glycerolphosphoesters, it hydrolyzes a variety of other phosphodiesters and displays low levels of activity against phosphomono- and triesters. Such a promiscuous nature may have assisted the apparent recent evolution of some binuclear metallohydrolases to deal with situations created by human intervention such as OP pesticides in the environment. OP pesticides were first used approximately 70 years ago, and therefore the enzymes that bacteria use to degrade them must have evolved very quickly on the evolutionary time scale. The promiscuous nature of enzymes such as GpdQ makes them ideal candidates for the application of directed evolution to produce new enzymes that can be used in bioremediation and against chemical warfare.

In this Account, we review the mechanisms employed by binuclear metallohydrolases and use PAP, the OP-degrading enzyme from Agrobacterium radiobacter (OPDA), and GpdQ as representative systems because they illustrate both the diversity and similarity of the reactions catalyzed by this family of enzymes. The majority of binuclear metallohydrolases utilize metal ionactivated water molecules as nucleophiles to initiate hydrolysis, while some, such as alkaline phosphatase, employ an intrinsic polar amino acid. Here we only focus on catalytic strategies applied by the former group.

\section{Introduction}

Enzymes catalyze most processes that constitute the chemistry of life, and life would be impossible without their remarkable ability to accelerate reaction rates. Hydrolytic enzymes constitute an important family of enzymes that are essential for recycling many of the molecules produced in cells. Among them, the binuclear metallohydrolases are a functionally diverse group whose members require two 


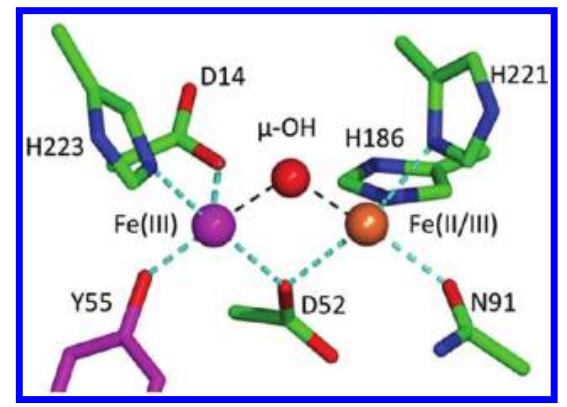

FIGURE 1. Binuclear metal ion center in PAP. PAPs have a $\mathrm{Fe}^{3+}$ in the "chromophoric" site, which forms a CT complex with an invariant tyrosine ligand, resulting in the characteristic purple color of the enzyme. Also shown is the metal ion bridging hydroxide, proposed to be one of two active hydrolysis-initiating nucleophiles (see text for details). Residue labels refer to the sequence of pig PAP.

metal ions in their active site to catalyze the hydrolysis of amides and esters of phosphoric acid. Figure 1 shows the metal ion coordination of a typical binuclear metalloenzyme. The imidazole side chains are the most common of the coordinating ligands followed by the acid side chains of aspartate and glutamate. Less frequent are ligands such as tyrosine. The metal ions are separated by about $3.5 \AA$ ( $\pm 0.5 \AA$ ) and are linked by bridging groups, which are generally hydroxides (i.e., $\mu$-hydroxides), side chains of amino acid residues, or a combination of both. ${ }^{1,2}$ The binuclear metal centers have been accommodated in a variety of protein folds, including four-helix bundles (e.g., arginase), TIM barrels (e.g., organophosphate-degrading enzyme from Agrobacterium radiobacter, OPDA), and $\alpha / \beta / \beta / \alpha$ folds (e.g., purple acid phosphatase, PAP, and organophosphate (OP)-degrading enzyme from Enterobacter aerogenes, GpdQ). A number of detailed reviews of the structures and functions of binclear metallohydrolases have been previously published, ${ }^{1,2}$ and this Account is intended to serve as an update of progress made in the past few years.

Binuclear metallohydrolases catalyze numerous vital reactions, and it is not surprising that they are potential targets of drugs against a wide variety of human disorders (e.g., osteoporosis, various cancers, antibiotic resistance, and erectile dysfunctions). ${ }^{1,2}$ These enzymes also tend to be promiscuous; they catalyze more than one reaction. For example, the GpdQ enzyme is part of a pathway that is used by bacteria to degrade glycerolphosphoesters, but it can also hydrolyze a variety of other phosphodiesters and displays low levels of activity against phosphomono- and triesters. ${ }^{3}$ This promiscuous nature may in part explain why binuclear metallohydrolases appear to have evolved in recent times to deal with situations created by man. For example, the organophosphate pesticides appeared in the environment about 70 years ago, so the enzymes that bacteria use to degrade them must have evolved very quickly in terms of evolutionary time. ${ }^{3,4}$ The promiscuous nature of these enzymes makes them ideal candidates for the application of directed evolution to produce new enzymes that can be used in bioremediation and as a defense against chemical warfare. In short, the binuclear metallohydrolases are a diverse set of enzymes that have the potential to be used in a variety of practical applications.

Binuclear metallohydrolases utilize a range of different metal ion combinations. ${ }^{1,2}$ The majority of these enzymes require two divalent metal ions for activity, and some are highly selective in terms of suitable metal ions, whereas others are functional with a range of different metal ions. Urease serves as an example of an enzyme with a specific requirement for $\mathrm{Ni}^{2+},{ }^{2}$ while GpdQ is catalytically active with a wide range of divalent metal ions. ${ }^{6}$ One member of the family of binuclear metallohydrolases, PAP, is unique in that it requires a heterovalent metal center of the type $\mathrm{Fe}^{3+}-\mathrm{M}^{2+}$ for catalytic activity (where $\mathrm{M}=\mathrm{Fe}, \mathrm{Zn}$, or $\mathrm{Mn}$ ). ${ }^{1,2}$ With respect to their proposed reaction mechanisms these enzymes can be separated into two distinct groups. One of them, represented by the di- $\mathrm{Zn}^{2+}$ enzyme alkaline phosphatase, employs an active site side chain (i.e., a serine residue) to initiate hydrolysis; in the process of the reaction a covalently linked reaction intermediate is formed. This reaction has been reviewed in great detail ${ }^{7}$ and will not be discussed further here. The majority of binuclear metallohydrolases utilize a solvent-derived hydroxide as the hydrolysis-initiating nucleophile. ${ }^{1,2}$ In most cases, this hydroxide is directly coordinated to one or both of the metal ions in the active site, but evidence has emerged that implicates a noncoordinated hydroxide in the outer coordination sphere as a possible alternative nucleophile (vide infra). ${ }^{2}$

Here, we present an Account of the mechanisms employed by binuclear metallohydrolases and use PAP and organophosphate-degrading enzymes as paradigms since they illustrate both the diversity and similarity of the reactions catalyzed by this family of enzymes. Other members, such as the metallo- $\beta$-lactamases (MBLs), enzymes that degrade most commonly used $\beta$-lactam antibiotics and thus present a major threat to global health, generally use similar mechanistic strategies to those discussed here, although it needs to be pointed out that in the case of MBLs alternative reaction schemes may be operational whereby only one metal ion is required for catalysis. ${ }^{8}$ Our choice to focus on PAP and organophosphate-degrading enzymes is also 


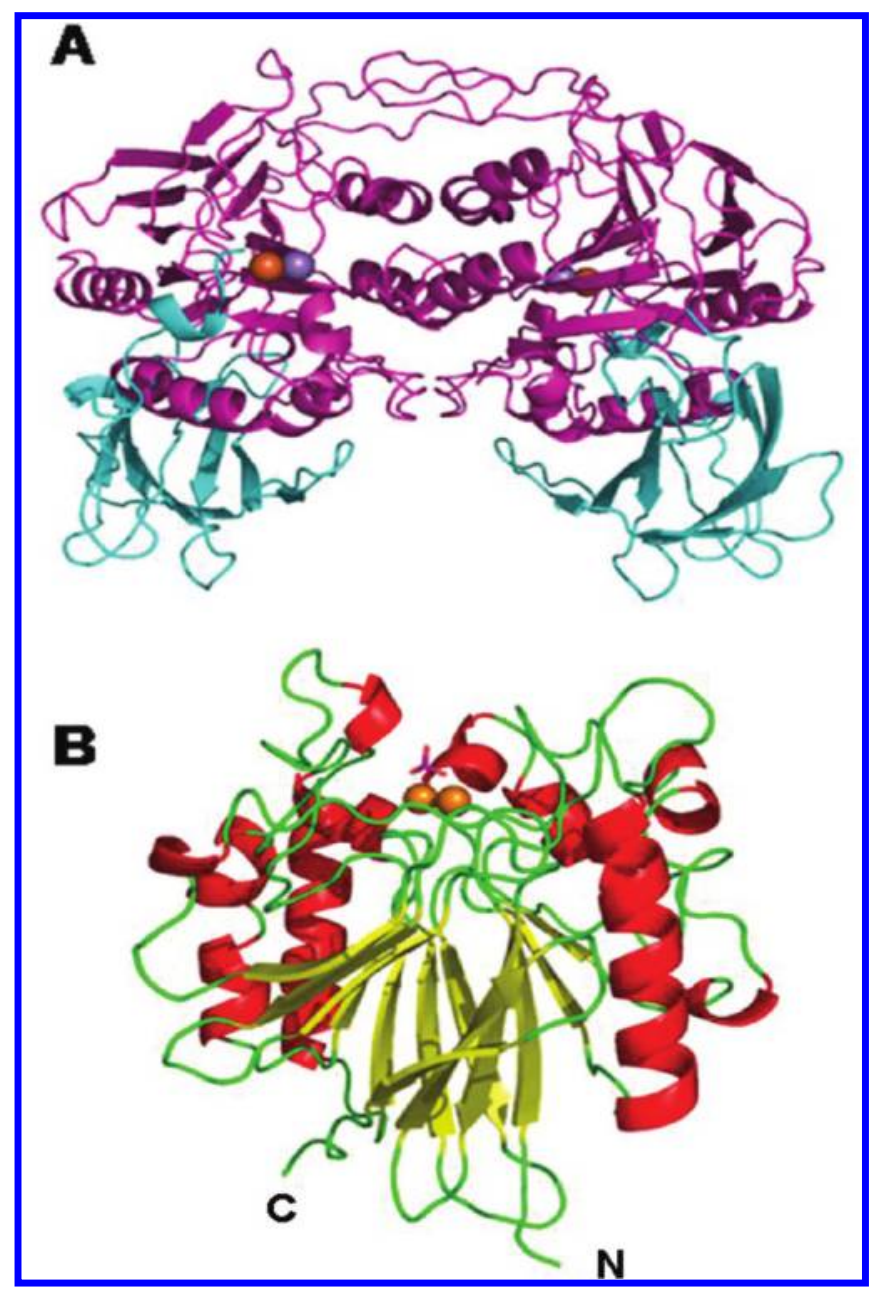

FIGURE 2. Overall structures of plant (A) and animal (B) PAPs. Most plant PAPs are homodimeric with $55 \mathrm{kDa}$ subunits. Each subunit has two domains, an $\mathrm{N}$-terminal without known function and a C-terminal that contains the active site. Animal PAPs are $35 \mathrm{kDa}$ monomers.

motivated by their established potential as drug targets and bioremediators, respectively, allowing us to illustrate the application of binuclear metallohydrolases in medicine and biotechnology.

\section{Purple Acid Phosphatases}

General Properties. PAPs are the only binuclear metallohydrolases known that require a heterovalent metal center for activity. ${ }^{1,2}$ The trivalent metal ion is invariably an $\mathrm{Fe}^{3+}$, although in vitro studies indicated that other trivalent metal ions $\left(\mathrm{Ga}^{3+}, \mathrm{Al}^{3+}\right)$ can reconstitute activity. ${ }^{2,9}$ In the case of a PAP from sweet potato, the $\mathrm{Fe}^{3+}$ could be replaced by $\mathrm{Mn}^{2+}$, leading to a $\mathrm{Mn}^{2+}-\mathrm{Mn}^{2+}$ derivative that operates most effectively at alkaline $\mathrm{pH}$ and is no longer purple. ${ }^{10}$ The origin of the purple color is a charge transfer (CT) interaction between the $\mathrm{Fe}^{3+}$ and a conserved tyrosine residue. ${ }^{11}$ The divalent metal ion is species-dependent and is $\mathrm{Fe}^{2+}$ in animals and $\mathrm{Zn}^{2+}$ or $\mathrm{Mn}^{2+}$ in plants (no bacterial PAP has yet been characterized). ${ }^{1,2}$ In animal PAPs, $\mathrm{Fe}^{2+}$ can be reversibly oxidized (reduction potential $\sim 340 \mathrm{mV}$ ), facilitating both an in vivo mechanism for the regulation of PAP activity and the provision for an alternative function, that is, peroxidation (Haber-Weiss-Fenton-type reaction). ${ }^{12,13}$

Apart from differences in their in vivo metal ion compositions, animal and plant PAPs can be distinguished by their molecular weights. Animal PAPs characterized to date are $35 \mathrm{kDa}$ monomers, and most plant PAPs are homodimeric with $55 \mathrm{kDa}$ subunits (Figure 2). ${ }^{2}$ However, mono- and heterodimeric plant PAPs have also been reported. ${ }^{2,14}$ The $35 \mathrm{kDa}$ animal PAPs and the $55 \mathrm{kDa}$ plant subunits share very little sequence homology $(<20 \%)^{2}$ However, sequence data analyses identified a putative PAP homologous to the $35 \mathrm{kDa}$ animal enzyme in plants. ${ }^{2}$ Similarly, a gene sequence encoding a putative human PAP homologous to the $55 \mathrm{kDa}$ plant isoform has been discovered. ${ }^{2}$ While very little is known about these alternative isoforms, their presence indicates that the function(s) of PAPs may be far more diverse than previously anticipated.

Overall and Active Site Structure. PAP crystal structures from several animal and plant sources have been reported (Figure 2). ${ }^{2,15-18}$ The plant enzymes consist of two domains; the C-terminal domain is similar to the structure of the animal enzyme and contains the active site while the $\mathrm{N}$-terminal domain has no known function. Despite low sequence homology between enzymes from different kingdoms, their catalytic sites are remarkably similar (Figure 1 ). ${ }^{2}$ In both plant and animal enzymes, the two metal centers are coordinated by seven invariant ligands, one aspartate, one tyrosine, and one histidine for $\mathrm{Fe}^{3+}$ and two histidines and one asparagine for the divalent metal ion, with an aspartate residue bridging the two ions. ${ }^{2,15-18}$ Water ligands are also present but their exact number may depend on experimental (i.e., crystallization) conditions and the source of the enzyme. ${ }^{2,17,18}$ A series of spectroscopic and kinetic measurements has furthermore demonstrated that the number of water ligands within a particular PAP may also depend on what substrate the enzyme utilizes, its metal ion composition, and the $\mathrm{pH}$ of the environment. ${ }^{2,10,11,18-20}$ Consequently, the molecular details of the reaction mechanism (i.e., the identity of the hydrolysis-initiating nucleophile) may also alter depending on experimental parameters.

Catalytic Mechanism. From a combination of crystallographic, kinetic, and spectroscopic data, an eight-step model for the catalytic mechanism employed by PAPs was developed (Figure 3). ${ }^{18}$ In the initial step, the substrate binds 


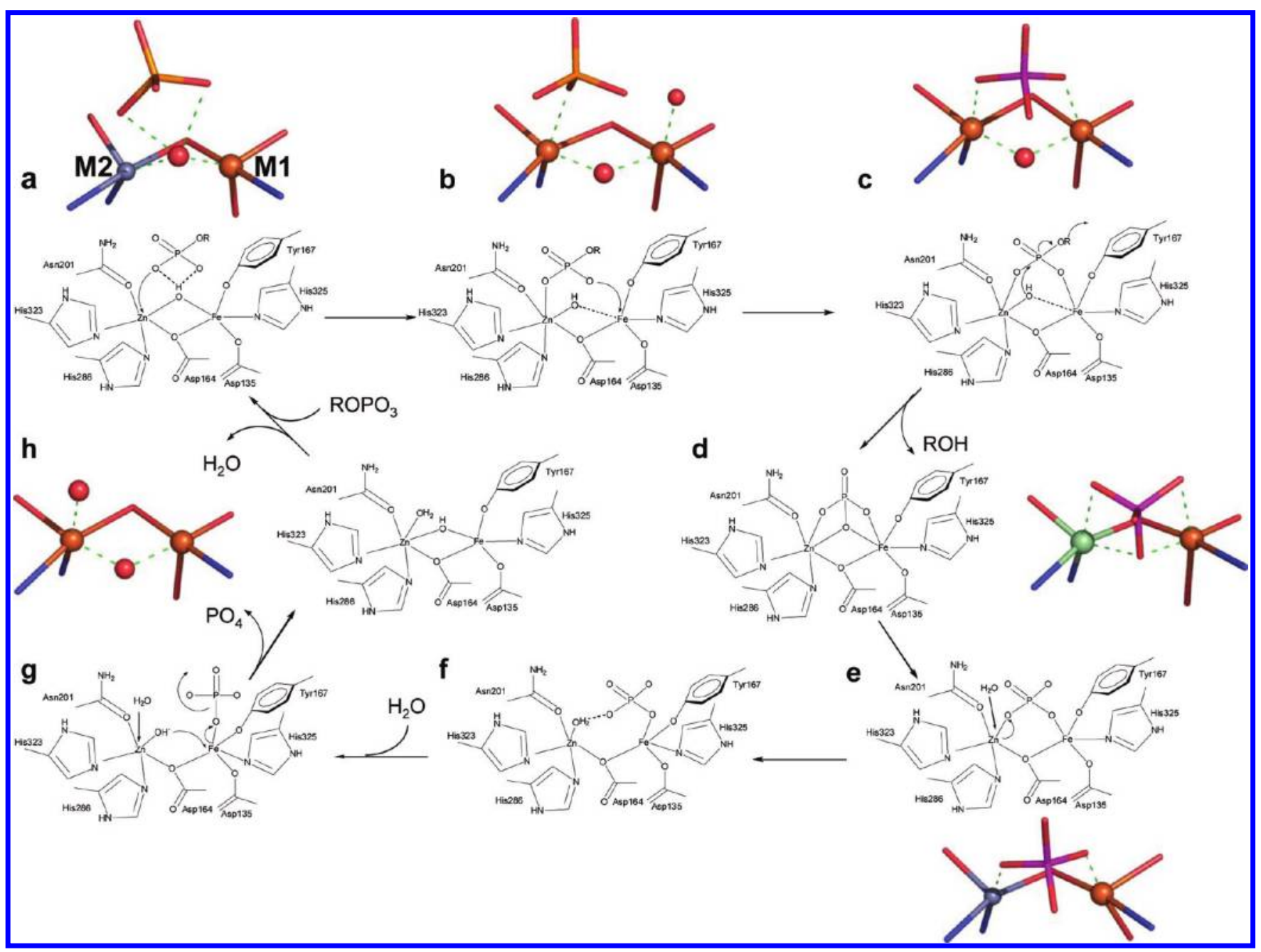

FIGURE 3. Proposed reaction mechanism for PAP-catalyzed hydrolysis. PAP and the substrate interact to form a precatalytic complex; subsequently, the substrate is rearranged to coordinate directly to at least one of the metal ions in the active site $(\mathrm{a}-\mathrm{c})$. Nucleophilic attack by either the $\mu$-hydroxide (as shown) or a noncoordinating hydroxide in the second coordination sphere is followed by the release of the leaving group, and the active site is returned to its resting state by the exchange of the bound phosphate group by two water molecules $(\mathrm{d}-\mathrm{h})$. Where available crystallographic snapshots (stick models) of relevant active site structures are also shown. ${ }^{18}$ (a) red kidney bean PAP-sulfate complex; (b) rat PAP-sulfate complex; (c) pig PAP-phosphate complex; (d) sweet potato PAP-phosphate complex, (e) red kidney bean PAP-phosphate complex, and (h) red kidney beanPAP. Adapted with permission from ref 18.

to the enzyme in the second coordination sphere, forming a precatalytic complex stabilized via an extensive hydrogen bond network. ${ }^{2,18,20}$ Following its initial binding, the substrate rearranges to be coordinated to the divalent metal ion, thus moving to a position that facilitates the nucleophilic attack by a solvent-derived hydroxide. The identity of this nucleophile has been debated extensively. ${ }^{2,9-11,15-20}$ The processive cleavage of the two ester bonds in a diester substrate indicated that PAP has two active nucleophiles (i.e., the product of the first reaction is not released but undergoes a second reaction; only then is the active site regenerated). ${ }^{19}$ One of them is the metal ion-bridging hydroxide $(u-\mathrm{OH})$, whose nucleophilicity is increased by a substrate-induced shift toward a "quasi-monodentate" position. ${ }^{2,11,18}$ The second nucleophilic hydroxide is not directly coordinated to the metal ions but instead is located in the outer coordination sphere, activated through hydrogen bonds to histidine residues that line the substrate binding pocket. ${ }^{20}$

Following the nucleophilic attack by a hydroxide and the concomitant ester hydrolysis of the substrate, the leaving group alcohol is released. The extent of bond breakage and formation in the transition state of this step varies considerably between different PAPs and different metal ion derivatives of a particular PAP, as evidenced by differences in their Brønsted correlations (i.e., the effect of the $p K_{a}$ values of the leaving group alcoholates on the catalytic rate), an observation that illustrates the effect of, in particular, the divalent 
metal ion in transition state formation. ${ }^{17}$ The second product of hydrolysis, the phosphate group, remains bound to the active site in either $\mathrm{a} \mu-1,3$ bridging or a tripodal $\left(\mu-\eta^{2}-\eta^{2}\right)$ geometry. ${ }^{17,18}$ The least understood step of the catalytic cycle is the regeneration of the resting state, which requires the removal of the phosphate group and, if the $\mu-\mathrm{OH}$ acted as nucleophile, the re-formation of this bridge. A plausible sequence is depicted in Figure 3 (steps $f-h$ ), where ligand exchange at the divalent metal site facilitates the incorporation of a water molecule that interacts with the $\mathrm{Fe}^{3+}$, thus regenerating the $\mu$-hydroxide bridge. The formation of the $\mu-\mathrm{OH}$ link is anticipated to weaken the $\mathrm{Fe}^{3+}$-phosphate bond, leading to the release of the phosphate group and the return of the active site to its resting state. ${ }^{9}$

Biological Role and Medicinal Significance. While PAPS act predominantly as hydrolases, they are bifunctional enzymes due to their ability to carry out peroxidations. ${ }^{12-14}$ Thus, their biological functions may be diverse. Suggested roles for PAPs include (i) iron transport, (ii) the generation of reactive oxygen species (ROS) as an immune response, (iii) energy metabolism, and (iv) bone resorption. ${ }^{2,13,21,22}$ Plant PAPs are furthermore also implicated in phosphate uptake and metabolism. ${ }^{2,14}$ Of particular clinical relevance is the observation of high PAP levels in animals at the resorptive interface formed between osteoclasts and bone, where PAP is required for active resorption. ${ }^{21}$ In osteoclasts, PAP dephosphorylates bone matrix proteins such as osteopontin. ${ }^{2,21}$ Furthermore, transgenic mice, in which osteoclasts overexpress and secrete PAP into the bone-resorptive space, display increased levels of resorption and display mild osteoporosis, while PAP knockout transgenic mice are osteopetrotic. ${ }^{21}$ Consequently, PAP has become a target for the development of antiosteoporotic chemotherapeutics. ${ }^{21}$

PAP is inhibited by simple anions such as fluoride $\left(\mathrm{F}^{-}\right)$ and oxyanions including molybdate $\left(\mathrm{MoO}_{4}{ }^{2-}\right)$, phosphate $\left(\mathrm{H}_{2} \mathrm{PO}_{4}{ }^{-} / \mathrm{HPO}_{4}{ }^{2-}\right)$, vanadate $\left(\mathrm{H}_{2} \mathrm{VO}_{4}{ }^{-}\right)$, and tungstate $\left(\mathrm{HWO}_{4}{ }^{-}\right)^{2}$, but their nonspecificity, size, overall charge, and lack of "drug-like" properties make them unsuitable as drug leads. In contrast, substrate mimics, such as phosphonates in which the cleavable $\mathrm{O}-\mathrm{P}$ bond has been replaced with a nonhydrolyzable $\mathrm{C}-\mathrm{P}$ bond, have led to the discovery of a number of promising leads. PAP inhibitors with potential for the development of antiosteoporotic drugs have recently been reviewed, ${ }^{23}$ and only some highlights are discussed here. Hydrophobic patches are located on the surface of PAPs close to the binuclear metal ion center, and this has been exploited in the design of potent inhibitors. ${ }^{24}$ A series of $\alpha$-alkoxy-substituted naphthalene-1ylmethylphosphonic acids in which the length of the alkyl chain

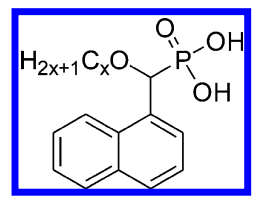

FIGURE 4. $\alpha$-Alkoxy-substituted naphthalene-1-ylmethylphosphonic acid derivatives. The aliphatic side chain increases both the potency and specificity of these inhibitors for PAP. ${ }^{23,24}$

was systematically altered was tested for their inhibitory effects on animal and plant PAP activity (Figure 4). The inhibitory effect depends on the length of the aliphatic chain, reaching a maximum potency with 14 carbon atoms ( $x=14$ in Figure 4$)$ and a measured $K_{\mathrm{i}}$ value of $\sim 200 \mathrm{nM}$ (unpublished results). The aliphatic side chain acts as an anchor, thus enhancing the binding affinity of the phosphonate derivative.

\section{Organophosphate-Degrading Enzymes}

General Properties. Synthetic organophosphate triesters (OPs) have revolutionized agriculture over the last 70 years. $^{3}$ However, these compounds are toxic (some have been used as nerve agents, for example, sarin or VX, which are declared as weapons of mass destruction). ${ }^{3,4}$ Furthermore, these compounds are not easily broken down; hence they accumulate in the environment. Some soil-dwelling bacteria have used environmental OP contamination to their advantage by evolving enzymes to degrade (hydrolyze) many of these compounds as a source of phosphorus for their metabolism. The most thoroughly characterized of these enzymes are the closely related phosphotriesterases ( $>90 \%$ sequence identity) from Flavobacterium sp ATCC 27551, Pseudomonas diminuta, and Agrobacterium radiobacter. ${ }^{3,4} \mathrm{~A}$ highly promiscuous phosphodiesterase from Enterobacter aerogenes, GpdQ, has also been shown to hydrolyze OPs, including EA2192, a highly toxic product of the degradation of the nerve agent $V X^{3,25}$ Thus, these enzymes have attracted increasing attention due to their ability to act as bioremediators. ${ }^{26}$ Here we will focus on the properties of the OP-degrading enzyme from A. radiobacter (OPDA) and GpdQ. Both enzymes are promiscuous with respect to the substrates they utilize but also in terms of the metal ions that are suitable to reconstitute catalytic activity. ${ }^{25-28}$ Atomic absorption spectroscopy and anomalous scattering measurements indicate that OPDA and GpdQ may contain $\mathrm{Fe}^{2+}-\mathrm{Zn}^{2+}$ centers in their native form, but this composition may be a reflection of natural abundance rather than catalytic optimization. ${ }^{6,29}$ In vitro studies demonstrated that the di-Co ${ }^{2+}$, di- $\mathrm{Mn}^{2+}$, and di-Cd ${ }^{2+}$ derivatives were significantly more reactive and efficient than the $\mathrm{Fe}^{2+}-\mathrm{Zn}^{2+}$ combination. 6,27,28 


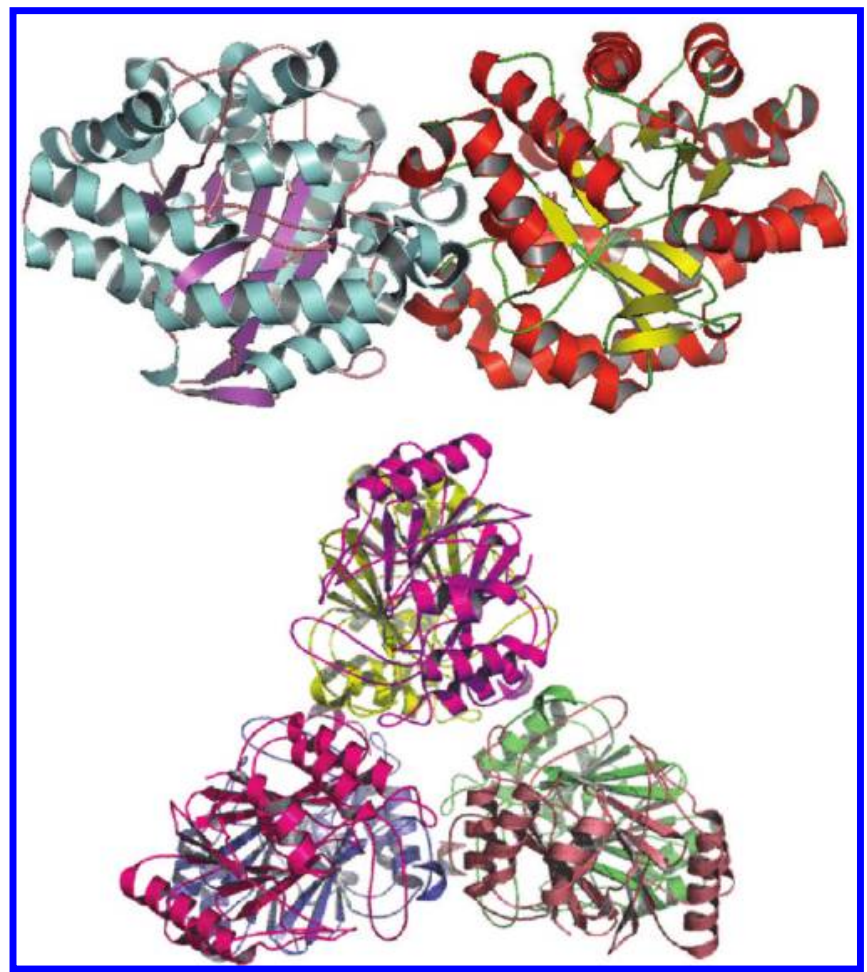

FIGURE 5. Overall structures of OPDA (top) and GpdQ (bottom). OPDA forms a homodimer, while GpdQ is homohexameric with a trimer of dimers oligomeric structure. . $^{3,27,28}$

Overall and Active Site Structure. The overall structure of OPDA is a homodimer; subunits fold to form a TIM barrel as is typical of amidohydrolases (Figure 5). ${ }^{3,28}$ GpdQ has a hexameric quaternary structure (a trimer of dimers) ${ }^{27}$ and has structural homology to PAPs (Figure 5). ${ }^{30}$ Six of the seven metal ion-coordinating amino acid side chains are identical in PAP and GpdQ (Figures 1 and 6). The $\alpha$-site in GpdQ corresponds to the $\mathrm{Fe}^{3+}$ site in PAP; the only difference is the replacement of His10 in GpdQ by a tyrosine in PAP. ${ }^{27}$ This tyrosine in PAP is involved in a CT interaction with $\mathrm{Fe}^{3+}$ (see above) leading to the characteristic color of PAP. The solvent-exposed $\beta$-site in GpdQ is identical to the divalent site of PAP; however, only in GpdQ does the asparagine ligand display coordination flexibility (see below) ${ }^{27}$ In combination with magnetic circular dichroism (MCD) studies, it was shown that two water molecules, one bridging the two metal ions and one bound terminally to the metal ion in the $\alpha$-site, complement the coordination spheres in the active site of GpdQ. ${ }^{27}$ In OPDA the binuclear metal center is located in the C-terminus of the $\beta$ barrel. ${ }^{28}$ Similar to GpdQ, a combination of crystallographic and MCD structural data indicates the presence of a five- and six-coordinate metal ion in the active site; however, while the $\alpha$-site has six ligands and the $\beta$-site five in GpdQ, the situation is reversed in OPDA (Figure 6). ${ }^{31}$

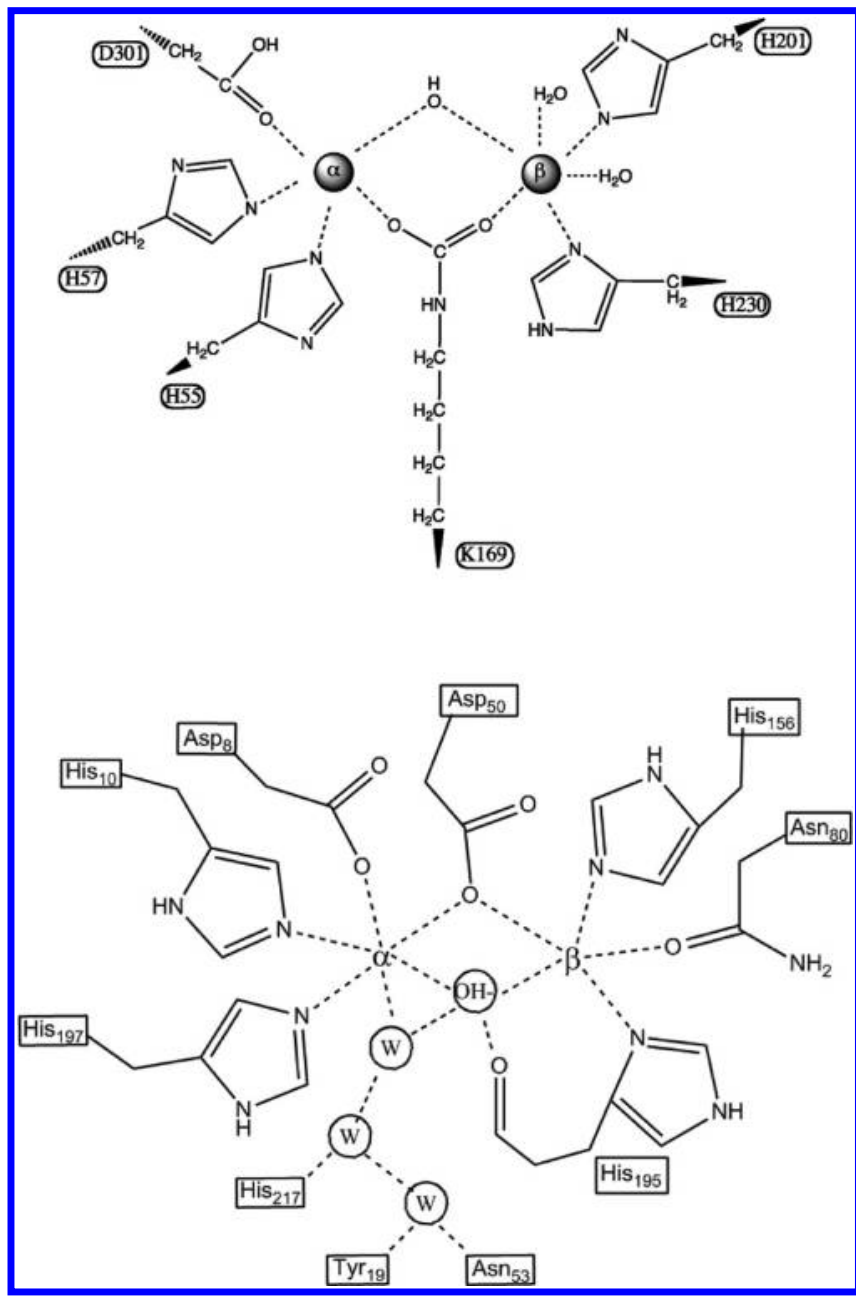

FIGURE 6. Schematic representations of the active structures of OPDA (top) and GpdQ (bottom). In OPDA the metal ions are bridged via a carboxylated lysine residue and a $\mu-\mathrm{OH}$. The active site of GpdQ is nearly identical to that of PAPs (Figure 1), with the exception of the His10Tyr substitution in GpdQ/PAP, respectively.

The more buried $\alpha$-site in OPDA is formed by two histidines and an aspartate, while the more solvent-exposed $\beta$-site also has two histidine ligands in addition to two terminal water molecules. The two metal ions are bridged by a carboxylated lysine and a hydroxide.

The active site of OPDA is virtually identical to that of its close relative, the OP-degrading enzyme from $P$. diminuta $(\mathrm{OPH}){ }^{3,4,28}$ Only three amino acid variations are observed in the substrate binding pocket (i.e., Arg254His, Tyr257His, and Phe272Leu in OPDA/OPH). Arg254 and Tyr257 in OPDA are part of an extensive hydrogen bond network that links the two metal ion centers via residues in the outer coordination sphere (Figure 7). ${ }^{28}$ Disruption of this hydrogen bond network through mutations leads to OPDA forms with catalytic properties and substrate specificities similar to that of $\mathrm{OPH}^{28}$ Thus, a small number of mutations leads to significant 


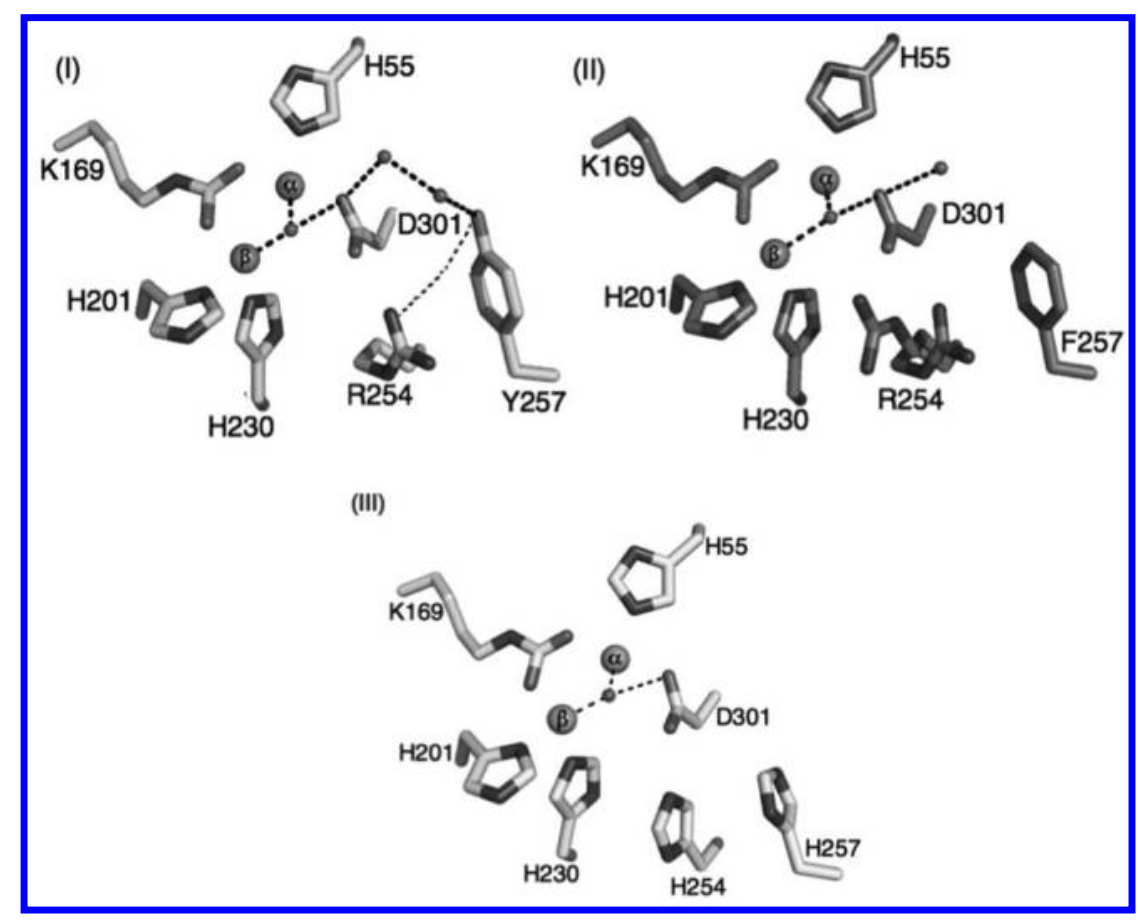

FIGURE 7. Comparison of the active site structures of OPDA (I), Tyr257Phe mutant OPDA (II), and OPH (III). The extensive hydrogen bonding network between $\mu$-OH, D301, Y257, and R254 in wild-type OPDA is disrupted in both the OPDA mutant and OPH. Reproduced from ref 28 . Copyright 2010 Biochemical Journal.

changes in the enzymatic properties of OP-degrading enzymes, a behavior that can be exploited to design a bioremediator with desired properties such as the preference for a particular type of substrate.

Catalytic Mechanism. The chemical mechanism for OP hydrolysis catalyzed by OPDA has been extensively studied by a number of enzymological, structural, and spectroscopic techniques, and evidence has emerged that indicates that this enzyme utilizes a flexible strategy that depends on its metal ion composition, the $\mathrm{pH}$ of the reaction, and possibly also the type of substrate. ${ }^{28,29,31-33}$ In Figure 8 , a recently proposed model for the mechanism is illustrated. In the initial phase of the catalytic cycle, the substrate binds in a monodentate fashion to the $\beta$-metal ion. At this stage, the substrate is primed for a nucleophilic attack by a metal ionbound hydroxide. The identity of this nucleophile has been subject to considerable debate; for $\mathrm{OPH}$, the $\mu$-hydroxide was proposed, while for OPDA, a hydroxide terminally bound to one of the metal ions was suggested. ${ }^{3,4}$ Due to their nearly identical active sites (only three amino acid differences in the substrate binding pocket; see above) a highly conserved reaction mechanism for the two enzymes may have been expected. Recent studies employing site-directed mutagenesis, however, demonstrated that in particular two of the three variable amino acids near the active site, Arg254 and Tyr257, play a significant role in modulating the reaction catalyzed by OPDA due to their incorporation into a hydrogen bond network that connects the substrate binding pocket to the metal ion binding sites (Figure 7). ${ }^{28}$ This hydrogen bond network is not present in $\mathrm{OPH}$, and when the network was disrupted in OPDA (through mutations), this enzyme became more similar to $\mathrm{OPH}$. Thus, a small number of amino acid differences may indeed account for the observed functional differences in these two OP-degrading enzymes. Furthermore, while most catalytic studies with $\mathrm{OPH}$ were carried out using $\mathrm{Zn}^{2+}, \mathrm{Cd}^{2+}$, or $\mathrm{Mn}^{2+}$ as metal ions, OPDA was mostly studied in its di$\mathrm{Co}^{2+}$ form. We were thus interested in studying the effect of metal ion replacements on the catalytic properties of OPDA. ${ }^{28}$ Analysis of the $\mathrm{pH}$ dependence of the turnover number $\left(k_{\text {cat }}\right)$ and catalytic efficiency $\left(k_{\text {cat }} / K_{\mathrm{m}}\right)$ for the di- $\mathrm{Zn}^{2+}$ and $\mathrm{di}-\mathrm{Cd}^{2+}$ derivatives of OPDA indicated that they may use a mechanism similar to that of $\mathrm{OPH}$, that is, a $\mu$-hydroxide as nucleophile (Scheme 2 in Figure 8). Similar to OPH (and PAP, see above), in di- $\mathrm{Zn}^{2+}$ and di-Cd ${ }^{2+}$ OPDA, substrate binding is suggested to trigger a shift of the $\mu$-hydroxide into a "quasimonodentate" position. Upon hydrolysis of the substrate and removal of the leaving group alcohol, the diester phosphate product remains bound to the active site in a $\mu-1,3$ mode, the only metal ion bridge apart from the carboxylated lysine residue. Regeneration of the active site occurs via ligand exchange whereby two water molecules 


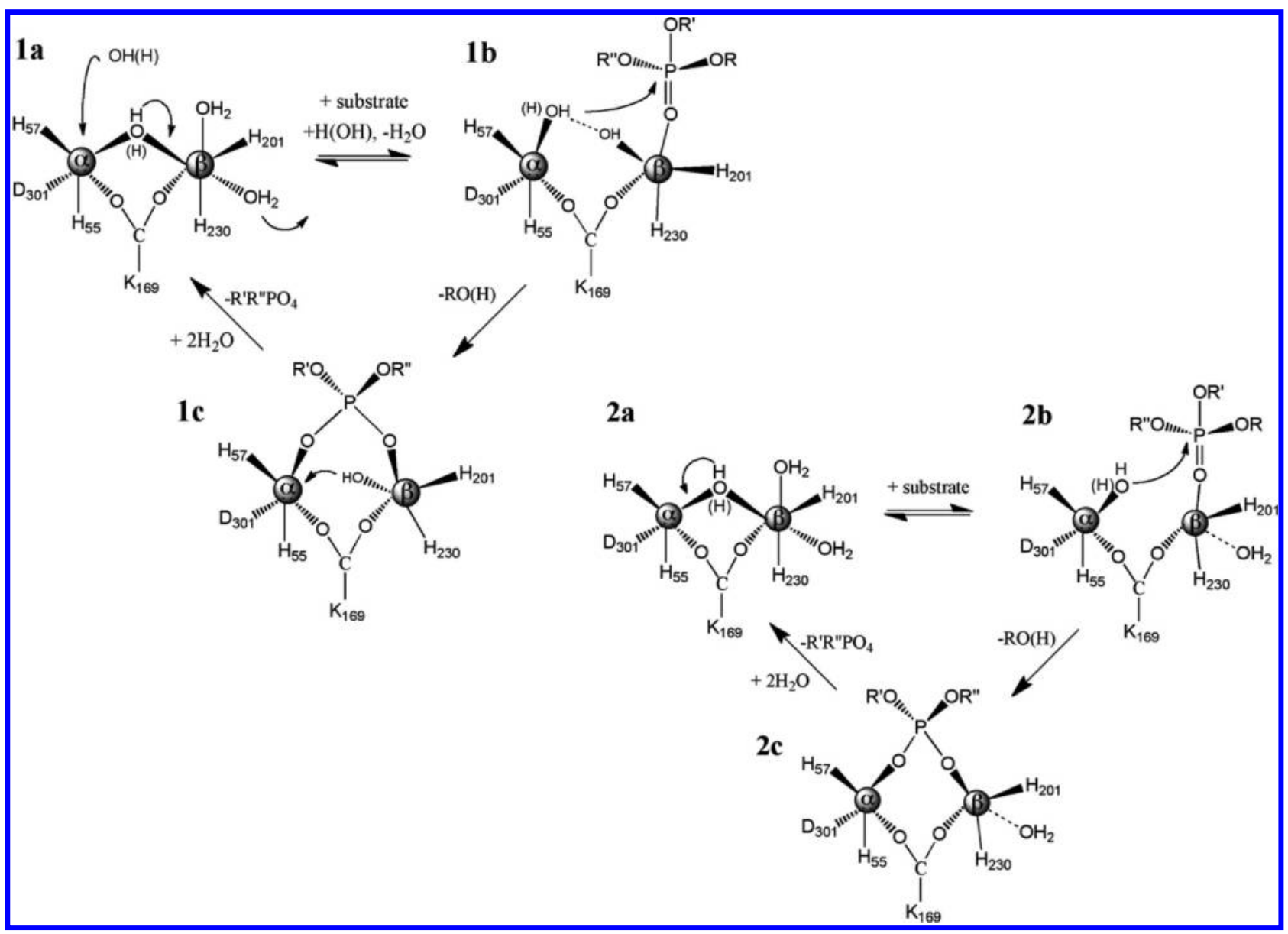

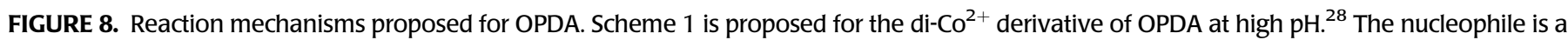
hydroxide terminally coordinated to the metal ion in the $\alpha$ site. Scheme 2 is proposed for the di-Co ${ }^{2+}$ derivative of OPDA at low pH, as well as for the di$\mathrm{Zn}^{2+}$ and di-Cd ${ }^{2+}$ derivatives and OPH (independent of $\left.\mathrm{pH}\right) .^{3,4,28}$ Reproduced from ref 31. Copyright 2011 Springer.

displace the diester product; one of the water molecules forms the metal ion bridge, while the other one completes the six-coordinate ligand sphere of the $\beta$-metal ion. The potential of the $\mu-\mathrm{OH}$ group to act as a nucleophile was recently visualized by a crystal structure in which $\mathrm{PO}_{4}{ }^{3-}$ was cocrystallized with OPDA. In the resulting complex, the tetraoxo anion was bound to the metal center in a tripodal arrangement, whereby two of its oxygen atoms bound monodentately to one metal ion each, while a third oxygen atom displaced the $\mu-\mathrm{OH}^{33}$ This tripodal arrangement was also observed in a phosphate complex of a sweet potato PAP and urease; in both systems, the $\mu-\mathrm{OH}$ is proposed to be the nucleophile of the reaction. ${ }^{17,34}$

However, metal ion replacement studies also demonstrated that different derivatives of OPDA may behave differently. This was exemplified by an investigation of the $\mathrm{pH}$ dependence of the catalytic parameters $\left(k_{\mathrm{cat}}\right.$ and $\left.k_{\mathrm{cat}} / K_{\mathrm{m}}\right)$ of the di-Co ${ }^{2+}$ derivative of OPDA. At low $\mathrm{pH}(<8-9)$, this derivative displays properties similar to those of the di- $\mathrm{Zn}^{2+}$ and di- $\mathrm{Cd}^{2+}$ forms (and thus OPH). However, $\mathrm{di}^{-\mathrm{Co}^{2+}}$ OPDA is most reactive at $\mathrm{pH}$ values above $10,{ }^{28}$ which is distinct from the behavior of other metal ion derivatives of this enzyme and $\mathrm{OPH}$. Under these conditions, $\mathrm{di}^{-\mathrm{CO}^{2+}} \mathrm{OPDA}$ is likely to use a terminally coordinated hydroxide as nucleophile, as illustrated in Scheme 1, Figure 8. Following initial substrate binding, structural rearrangements also lead to a shift of the $\mu-\mathrm{OH}$ group, in this case toward the $\beta$-metal ion. This shift provides a vacant coordination for a water molecule in the coordination sphere of the $\alpha$ metal ion. The nucleophilicity of this water is enhanced by hydrogen bond interactions with the "quasi-monodentate" hydroxide. Following hydrolysis by this terminal nucleophile, the diester phosphate group also remains bound to the active site in a $\mu-1,3$ mode, but a hydroxide in a bridging position is still present, as observed in some crystal structures of OPDA-product complexes. ${ }^{32}$

Due to the similarity of their active site structures it was anticipated that GpdQ employs a mechanism closely related 


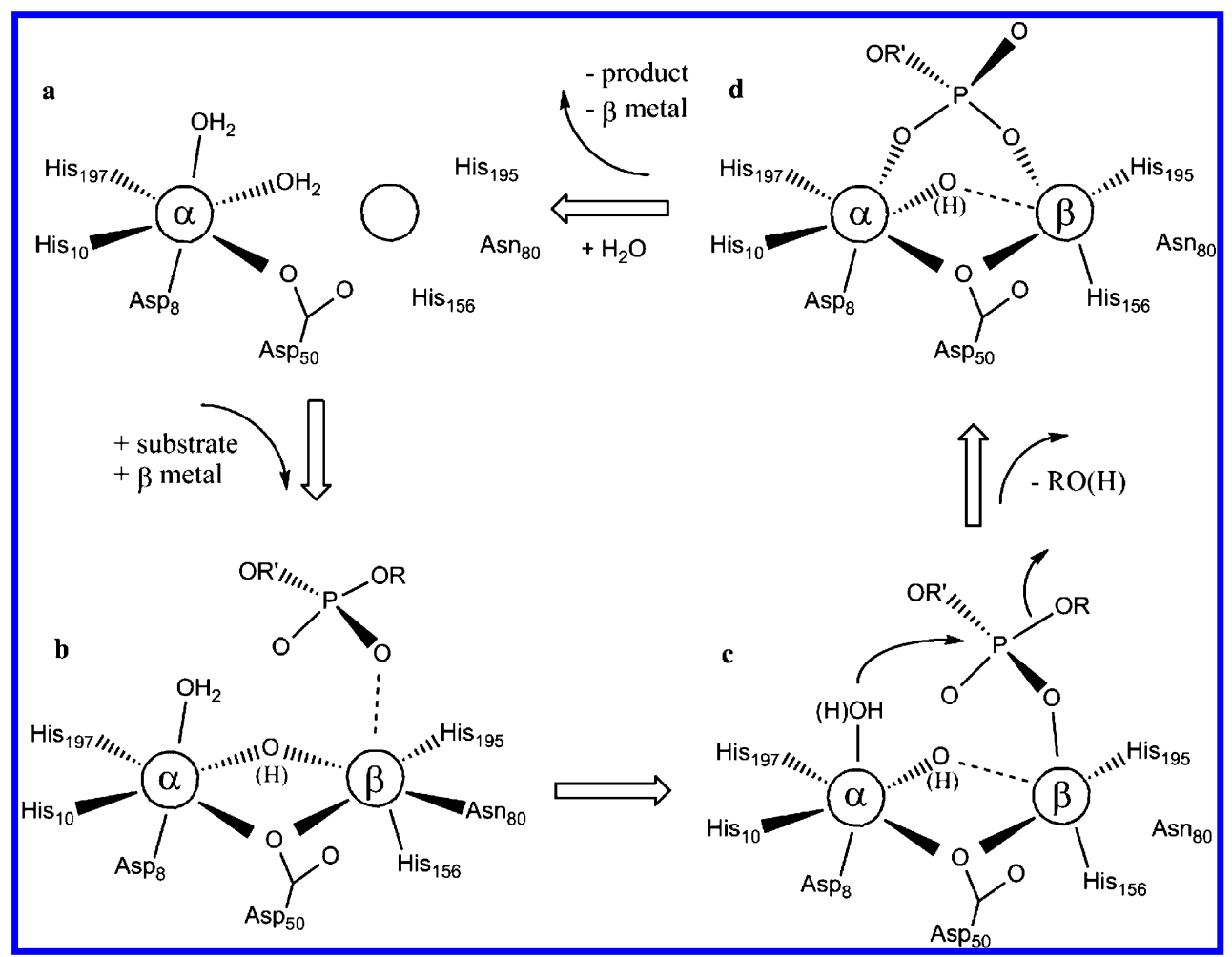

FIGURE 9. Reaction mechanism proposed for GpdQ. The enzyme is only active in the presence of a substrate (substrate-promoted activation), a regulatory mechanism that limits nonbeneficial reactions catalyzed by this very promiscuous hydrolase. ${ }^{27,35-37}$ Reproduced from ref 27 . Copyright 2008 American Chemical Society.

to that of PAP (Figures 1 and 6). However, it has recently emerged that GpdQ differs significantly from PAP in terms of the reaction mechanism it employs (Figure 9). In its resting state, the enzyme is predominantly mononuclear and catalytically inactive. ${ }^{27}$ The $\beta$-site has a low affinity for metal ions, but the addition of substrate (or a substrate analogue) enhances its affinity, leading to the formation of a catalytically active binuclear metal center. The enzyme remains catalytically fully active until the substrate is depleted. ${ }^{35}$ This regulation of the catalytic activity may be a built-in safety mechanism to prevent deleterious side reactions that may occur due to the substrate promiscuity displayed by GpdQ. GpdQ also displays coordination flexibility in the $\beta$-site; ligand Asn80 (Figures 6 and 9) assists in initial metal ion binding, however, upon formation of the enzyme-substrate complex its bond to the metal ion is broken. ${ }^{27,35}$ An additional difference between the two enzymes is that PAP is able to hydrolyze the two ester bonds of a diester substrate in a processive manner (see above), while GpdQ acts in sequential manner whereby the product of the first reaction is released and a new enzyme-substrate complex needs to be formed. ${ }^{19,36}$ The reaction mechanism that emerged for GpdQ is illustrated in Figure 9. Substrate binding in the vicinity of the active site triggers a conformational change that increases the affinity of the $\beta$-site for metal ions. Upon the formation of a binuclear center, the substrate rearranges to coordinate directly to the metal ion in the $\beta$-site. This interaction leads to a bond break between this metal and Asn80. In this form, GpdQ is fully active and remains so as long as substrate is available. The identity of the nucleophile is again subject to debate, and similar to OPDA it is likely that different metal ion derivatives may also prefer a different nucleophile, either a $\mu$-OH or terminally bound hydroxide. ${ }^{6,27,35-37}$ Hence, both OPDA and GpdQ reveal flexibility with respect to their employed mechanistic strategies, a fact that may be advantageous for their rapid adaptation toward new environmental challenges.

Significance in Biotechnology and Medicine. Unlike other binuclear metallohydrolases such as PAPs, OP-degrading enzymes may not have a distinct biological function in their host organisms other than providing an advantage in survival in an environment that has been altered through human intervention. Since the introduction of OPs, the use of pesticides has increased dramatically, and currently the global market is worth about 30 billion dollars annually. 3,38 The concomitant expansion of agriculture has not been without a cost. OPs are toxic and stable and may be retained in the environment for long periods, causing the death of 
nontargeted insects and some higher organisms. ${ }^{38}$ Hundreds of thousands of people suffer from OP poisoning each year. ${ }^{39}$ While chemical treatments for OP poisoning exist, they generally act slowly. In contrast, recent trials with animals have shown that OPDA can quickly clear most of the pesticides in the blood of rats. ${ }^{40}$ Furthermore, this enzyme is already part of a commercial product (Landguard) that has been demonstrated to be efficient in removing OP pesticides from contaminated environment. ${ }^{26}$ Current limitations are the stability of the enzyme in an environmental setting, as well as its catalytic efficiency toward commonly used OPs. Attempts to overcome these limitations are currently being made using directed evolution methods; it is anticipated that in the near future bioremediation by OPdegrading enzymes will become a major contributor to solving pesticide contaminations that lead to environmental pollution and human poisoning.

\section{Final Comments}

Binuclear metallohydrolases are a large and growing family of enzymes that are involved in a broad range of metabolic functions. In this Account, we focused on three specific examples to illustrate their scope of functions and modes of action. However, the members of this enzyme family are functionally often only distinguished by their preference for particular substrates and employ similar complex mechanistic strategies to carry out a simple chemical reaction, the cleavage of ester and amide bonds. In some cases, enzymes with diverse biological functions may have evolved from a common ancestral protein. An example is a group of OPdegrading enzymes recently identified in bacterial strains from contaminated soil in China. ${ }^{3}$ Although the primary function of these enzymes is to hydrolyze OPs, their overall structure is more closely related to $\beta$-lactam-degrading MBLs than to OPDA or GpdQ. In other cases, for instance, OPDA and $\mathrm{GpdQ}$, enzymes evolved from different ancestors to acquire similar functions, a process that may have been triggered by environmental pressures.

Major advances have been made in our understanding of how binuclear metallohydrolases work and what roles in biology they play. While some aspects of their mechanisms at the molecular levels await further investigations, the garnering of the great potential of these enzymes in a multitude of applications is a future challenge that awaits the bioinorganic chemistry and biotechnology communities.

The support of the Australian Research Council and the National Health and Medical Research Council for funding is kindly appreciated. N.M. gratefully acknowledges the receipt of a SFIPIYRA Fellowship.

\section{BIOGRAPHICAL INFORMATION}

Gerhard Schenk currently holds joint professorial appointments in the School of Chemistry and Molecular Biosciences at the University of Queensland, Brisbane, Australia, and the Department of Chemistry at the National University of Ireland-Maynooth, Ireland. Current research is related to the investigation of the mechanism of binuclear metalloenzymes and related biomimetics.

Nataša Mitić was awarded a President of Ireland Young Researcher Award (PIYRA) from the Science Foundation Ireland (SFI) in 2010 and holds a position in the Department of Chemistry at the National University of Ireland-Maynooth (NUIM). Her current research interests are focused on the structure and function of antibiotic-degrading metallo- $\beta$-lactamases.

Lawrence R. Gahan is a Professor of Chemistry in the School of Chemistry and Molecular Biosciences at the University of Queensland, Brisbane, Australia. Current research is related to the design of synthetic and functional models for the active site of metalloenzymes.

David L. Ollis is a Professor of Chemistry in the Research School of Chemistry at the Australian National University, Canberra, Australia. Current research interests include directed evolution, with binuclear metalloenzymes being the subject of much of his work.

Ross P. McGeary is an Associate Professor at the University of Queensland. He holds joint appointments in the School of Chemistry and Molecular Biosciences and the School of Pharmacy. His main research interests are the design of new synthetic methodologies and the synthesis of inhibitors of binuclear metalloenzymes.

Luke W. Guddat is an Associate Professor in the School of Chemistry and Molecular Biosciences at the University of Queensland, Brisbane, Australia. The major focus of his research is to use protein X-ray crystallography in rational structure-based drug design.

\section{FOOTNOTES}

${ }^{*}$ To whom correspondence should be addressed. Telephone: ++61733654144 . Fax: ++61 73365 4273. E-mail: schenk@uq.edu.au.

The authors declare no competing financial interest.

\section{REFERENCES}

1 Wilcox, D. E. Binuclear Metallohydrolases. Chem. Rev. 1996, 96, 2435-2458.

2 Mitić, N.; Smith, S. J.; Neves, A.; Guddat, L. W.; Gahan, L. R.; Schenk, G. The Catalytic Mechanism of Binuclear Metallohydrolases. Chem. Rev. 2006, 106, 3338-3363.

3 Ely, F.; Foo, J.-L.; Jackson, C. J.; Gahan, L. R.; Ollis, D. L.; Schenk, G. Enzymatic Bioremediation: Organophosphate Degradation by Binuclear Metallo-hydrolases. Curr. Top. Biochem. Res. 2007, 9, 63-78.

4 Raushel, F. M. Bacterial Detoxification of Organophosphate Nerve Agents. Curr. Opin. Microbiol. 2002, 5, 288-295.

5 Zambelli, B.; Musiani, F.; Benini, S.; Ciurli, S. Chemistry of $\mathrm{Ni}^{2+}$ in Urease: Sensing, Trafficking, and Catalysis. Acc. Chem. Res. 2011, 44, 520-530.

6 Daumann, L. J.; McCarthy, B. Y.; Hadler, K. S.; Murray, T.; Gahan, L. R.; Larrabee, J. A.; Ollis, D. L.; Schenk, G. Promiscuity Comes at a Price: Catalytic Versatility vs Efficiency. Biochim. Biophys. Acta 201210.1016/j.bbapap.2012.02.004.

7 O'Brien, P. J.; Herschlag, D. Alkaline Phosphatase Revisited: Hydrolysis of Alkyl Phosphates. Biochemistry 2002, 41, 3207-3225.

8 Crowder, M. W.; Spencer, J.; Vila, A. J. Metallo- $\beta$-lactamases: Novel Weaponry for Antibiotic Resistance in Bacteria. Acc. Chem. Res. 2006, 39, 721-728. 
9 Smith, S. J.; Casellato, A.; Hadler, K. S.; Mitić, N.; Riley, M. J.; Bortoluzzi, A. J.; Szpoganicz, B.; Schenk, G.; Neves, A.; Gahan, L. R. The Reaction Mechanism of Binuclear Metallohydrolases: The Catalytic Role of the Metal lons. J. Biol. Inorg. Chem. 2007, 12, 1207-1220.

10 Mitić, N.; Noble, C. J.; Gahan, L. R.; Hanson, G. R.; Schenk, G. Metal Ion Mutagenesis Conversion of a Purple Acid Phosphatase from Sweet Potato to a Neutral Phosphatase with the Formation of an Unprecedented Catalytically Competent Mn" ${ }^{\prime \prime} n^{\|}$Active Site. J. Am. Chem. Soc. 2009, 131, 8173-8179.

11 Yang, Y.-S.; McCormick, J. M.; Solomon, E. I. Circular Dichroism and Magnetic Circular Dichroism Studies of the Mixed-Valence Binuclear Non-Heme Iron Active Site in Uteroferrin and Its Anion Complexes. J. Am. Chem. Soc. 1997, 119, 11832-11842.

12 Bernhardt, P. V.; Schenk, G.; Wilson, G. J. Direct Electrochemistry of Porcine Purple Acid Phosphatase (Uteroferrin). Biochemistry 2004, 43, 10387-10392.

13 Raisänen, S. R.; Alatalo, S. L.; Ylipahkala, H.; Halleen, J. M.; Cassady, A. I.; Hume, D. A.; Väänänen, K. Macrophages Overexpressing Tartrate-Resistant Acid Phosphatase Show Altered Profile of Free Radical Production and Enhanced Capacity of Bacterial Killing. Biochem. Biophvs. Res. Commun. 2005, 331, 120-126.

14 Bozzo, G. G.; Raghothama, K. G.; Plaxton, W. C. Structural and Kinetic Properties of a Novel Purple Acid Phosphatase from Phosphate-Starved Tomato (Lycopersicon esculentum) Cell Cultures. Biochem. J. 2004, 377, 419-428.

15 Sträter, N.; Klabunde, T.; Tucker, P.; Witzel, H.; Krebs, B. Crystal Structure of a Purple Acid Phosphatase Containing a Dinuclear Fe(III)-Zn(II) Active Site. Science 1995, 268, 14891492.

16 Guddat, L. W.; McAlpine, A. S.; Hume, D.; Hamilton, S.; de Jersey, J.; Martin, J. L. Crystal Structure of Mammalian Purple Acid Phosphatase. Structure 1999, 7, 757-767.

17 Schenk, G.; Gahan, L. R.; Carrington, L. E.; Mitić, N.; Valizadeh, M.; Hamilton, S. E.; de Jersey, J.; Guddat, L. W. Phosphate Forms an Unusual Tripodal Complex with the Fe-Mn Center of Sweet Potato Purple Acid Phosphatase. Proc. Natt. Acad. Sci. U.S.A. 2005, 102, 273-278.

18 Schenk, G.; Elliott, T. W.; Leung, E. W. W.; Mitić, N.; Carrington, L. E.; Gahan, L. R.; Guddat, L. W. Snapshots of the Reaction Mechanism of Purple Acid Phosphatase-Catalyzed Hydrolysis. BMC Struct. Biol. 2008, 8, 6.

19 Cox, R. S.; Schenk, G.; Mitić, N.; Gahan, L. R.; Hengge, A. C. Diesterase Activity and Substrate Binding in Purple Acid Phosphatases. J. Am. Chem. Soc. 2007, 129, 9550-9551.

20 Mitić, N.; Hadler, K. S.; Gahan, L. R.; Hengge, A. C.; Schenk, G. The Divalent Metal lon in the Active Site of Uteroferrin Modulates Substrate Binding and Catalysis. J. Am. Chem. Soc. 2010, 132, 7049-7054

21 Oddie, G. W.; Schenk, G.; Angel, N. Z.; Walsh, N.; Guddat, L. W.; de Jersey, J.; Cassady, A. I.; Hamilton, S. E.; Hume, D. A. Structure, Function and Regulation of Tartrate-Resistant Acid Phosphatase. Bone 2000, 27, 575-584.

22 Mitić, N.; Valizadeh, M.; Leung, E. W. W.; de Jersey, J.; Hamilton, S.; Hume, D. A.; Cassady, A. I.; Schenk, G. Human Tartrate-Resistant Acid Phosphatase Becomes an Effective ATPase upon Proteolytic Activation. Arch. Biochem. Biophvs. 2005, 439, 154-164.

23 Vella, P.; McGeary, R. P.; Gahan, L. R.; Schenk, G. Tartrate-Resistant Acid Phosphatases, A Target for Novel Anti-Osteoporotic Chemotherapeutics. Curr. Enz. Inhib. 2010, 6, 118-129.

24 McGeary, R. P.; Vella, P.; Mak, J. Y. W.; Guddat, L. W.; Schenk, G. Inhibition of Purple Acid Phosphatase with $\alpha$-Alkoxynaphthylmethylphosphonic Acids. Bioorg. Med. Chem. Lett. 2009, 19, 163-166.

25 Ghanem, E.; Li, Y.; Xu, C.; Raushel, F. M. Characterization of a Phosphodiesterase Capable of Hydrolyzing EA 2192, the Most Toxic Degradation Product of the Nerve Agent VX. Biochemistry 2007, 46, 9032-9040.
26 Horne, l.; Qiu, X.; Ollis, D. L.; Russell, R. J.; Oakeshott, J. G. Functional Effects of Amino Acid Substitutions within the Large Binding Pocket of the Phosphotriesterase OpdA from Agrobacterium sp. P230. FEMS Microbiol. Lett. 2006, 259, 187-194.

27 Hadler, K. S.; Tanifum, E. A.; Yip, S.; Mitić, N.; Guddat, L. W.; Jackson, C. J.; Gahan, L. R.; Nguyen, K.; Carr, P. D.; Ollis, D. L.; Hengge, A. C.; Larrabee, J. A.; Schenk, G. SubstratePromoted Formation of a Catalytically Competent Binuclear Center and Regulation of Reactivity in Glycerophosphodiesterase from Enterobacter aerogenes. J. Am. Chem. Soc. 2008, 130, 14129-14138.

28 Ely, F.; Hadler, K. S.; Gahan, L. R.; Guddat, L. W.; Ollis, D. L.; Schenk, G. Catalytic Mechanism of the Hydrolytic Reaction Catalyzed by an Organophosphate-Degrading Enzyme from Agrobacterium radiobacter. Biochem. J. 2010, 432, 565-573.

29 Jackson, C. J.; Carr, P. D.; Kim, H.-K.; Liu, J.-W.; Herrald, P.; Mitić, N.; Schenk, G.; Smith, C. A.; Ollis, D. L. Anomalous Scattering Analysis of Agrobacterium radiobacter Phosphotriesterase: The Prominent Role of Iron in the Heterobinuclear Active Site. Biochem. J. 2006, 397, 501-508.

30 Hadler, K. S.; Huber, T.; Cassady, I. A.; Weber, J.; Robinson, J.; Burrows, A.; Kelly, G.; Guddat, L. W.; Hume, D.; Schenk, G.; Flanagan, J. Identification of a Non-purple TartrateResistant Acid Phosphatase: An Evolutionary Link to Ser/Thr Protein Phosphatases? BMC Res. Notes 2008, 1, No. 78.

31 Ely, F.; Hadler, K. S.; Mitić, N.; Gahan, L. R.; Ollis, D. L.; Larrabee, J. A.; Schenk, G. Electronic and Geometric Structure of the Organophosphate-Degrading Enzyme from Agrobacterium radiobacter. J. Biol. Inorg. Chem. 2011, 16, 777-787.

32 Jackson, C. J.; Foo, J.-L.; Kim, H.-K.; Carr, P. D.; Liu, J.-W.; Salem, G.; Ollis, D. L. In Crystallo Capture of a Michaelis Complex and Product-Binding Modes of a Bacterial Phosphotriesterase. J. Mol. Biol. 2008, 375, 1189-1196.

33 Ely, F.; Pedroso, M. M.; Gahan, L. R.; Ollis, D. L.; Guddat, L. W.; Schenk, G. PhosphateBound Structure of an Organophosphate-Degrading Enzyme from Agrobacterium radiobacter: Comparison with Other Binuclear Metallohydrolases. J. Inorg. Biochem. 2012, 106, 19-22.

34 Benini, S.; Rypniewski, W. R.; Wilson, K. S.; Ciurli, S.; Mangani, S. Structure-Based Rationalization of Urease Inhibition by Phosphate: Novel Insights into the Enzyme Mechanism. J. Biol. Inorg. Chem. 2001, 6, 778-790.

35 Hadler, K. S.; Mitić, N.; Ely, F.; Hanson, G. R.; Gahan, L. R.; Larrabee, J. A.; Ollis, D. L.; Schenk, G. Structural Flexibility Enhances the Reactivity of the Bioremediator Glycerophosphodiesterase by Fine-Tuning Its Mechanism of Hydrolysis. J. Am. Chem. Soc. 2009, 131, 11900-11908

36 Hadler, K. S.; Gahan, L. R.; Ollis, D. L.; Schenk, G. The Bioremediator Glycerophosphodiesterase Employs a Non-Processive Mechanism for Hydrolysis. J. Inorg. Biochem. 2010, 104, 211-213.

37 Hadler, K. S.; Mitić, N.; Yip, S.H.-C.; Gahan, L. R.; Ollis, D. L.; Schenk, G.; Larrabee, J. A. Electronic Structure Analysis of the Binuclear Metal Center in the Bioremediator Glycerophosphodiesterase (GpdQ) from Enterobacter aerogenes. Inorq. Chem. 2010, 49, 2727 2734.

38 Costa, L. G. Current Issues in Organophosphate Toxicology. Clin. Chim. Acta 2006, 366, $1-13$.

39 Eddleston, M.; Karalliedde, L.; Buckley, N.; Fernando, R.; Hutchinson, G.; Isbister, G.; Konradsen, F.; Murray, D.; Piola, J. C.; Senanayake, N.; Sheriff, R.; Singh, S.; Siwach, S. B.; Smit, L. Pesticide Poisoning in the Developing World-a Minimum Pesticides List. Lancet 2002, 360, 1163-1167.

40 Bird, S. B.; Dawson, A.; Ollis, D. L. Enzymes and Bioscavengers for Prophylaxis and Treatment of Organophosphate Poisoning. Front. Biosci. 2010, S2, 209-220. 